\title{
Gene targeting to the uteroplacental circulation of pregnant guinea pigs
}

Vedanta Mehta $\mathrm{PhD}^{1,2}$, Keren Ofir $\mathrm{MD}^{1}$, Anna Swanson $\mathrm{PhD}^{1}$, Ewa Kloczko BSc${ }^{1}$, Michael Boyd $\mathrm{MSc}^{3}$, Hannah Barker MSc ${ }^{3}$, Adnan Avdic-Belltheus MSc ${ }^{3}$, John Martin $\mathrm{MD}^{2}$, Ian Zachary $\mathrm{PhD}^{2}$, Donald Peebles MD FRCOG ${ }^{1}$, Anna L David PhD MRCOG ${ }^{1}$

${ }^{1}$ Institute for Women's Health, University College London, London, UK

${ }^{2}$ Centre for Cardiovascular Biology and Medicine, University College London, London, UK

${ }^{3}$ BSU, Royal Veterinary College, London, UK

Corresponding author: Dr Vedanta Mehta,

Centre for Cardiovascular Biology and Medicine,

Division of Medicine, UCL

Rayne Building

5 University Street,

London WC1E 6JF

v.mehta@ucl.ac.uk

\section{Keywords:}

Prenatal gene therapy, uteroplacental circulation, guinea pig, adenovirus vector, pluronic gel, VEGF-A 165 


\begin{abstract}
Our study aimed to target adenoviral gene therapy to the utero-placental circulation of pregnant guinea pigs in order to develop a novel therapy for fetal growth restriction. Four methods of delivery of an adenovirus encoding $\beta$-galactosidase (Ad.LacZ) were evaluated: intravascular injection using phosphate buffered saline (PBS) into (1) uterine artery or (2) internal iliac artery, or external administration in (3) PBS or (4) Pluronic F-127 gel (Sigma Aldrich). Post mortem examination was performed 4-7 days after gene transfer. Tissue transduction was assessed by Xgal histochemistry and ELISA. External vascular application of the adenovirus vector in combination with pluronic gel had $91.7 \%$ success rate in terms of administration (85\% maternal survival) and gave the best results for maternal/fetal survival and local transduction efficiency without any spread to maternal or fetal tissues. This study suggests an optimal method of gene delivery to the uterine arteries of a small rodent for pre-clinical studies.
\end{abstract}




\section{Introduction}

Fetal Growth Restriction (FGR) is an obstetric complication in which the fetus does not achieve its genetically determined growth potential. Pregnancies complicated by FGR have an increased rate of perinatal complications and mortality, and long term there is an increased risk of cardiovascular and neurological disorders ${ }^{1-6}$. It has been established that reduced uteroplacental perfusion can cause FGR and to the best of our knowledge, treatments or management strategies remain elusive.

We have previously shown that in pregnant sheep, there was a significant increase in uterine blood flow (UBF) for up to one month after injection of adenovirus vectors containing the VEGF$\mathrm{A}_{165}$ gene when compared with uterine arteries (UtAs) injected with a control adenovirus containing the $\beta$-galactosidase gene $\mathrm{e}^{7,8}$. Moreover, we have demonstrated a reduction in UtA contractility and adventitial neovascularization 30-45 days after local administration of Ad.VEGF-A 165 to the UtAs of pregnant sheep ${ }^{7,8}$. There was no undesirable vector expression in other maternal/fetal tissues as determined by semi-nested RT-PCR. Recent data from sheep pregnancies affected by fetal growth restriction demonstrates that mid-gestation maternal UtA injection of Ad.VEGF-A 165 vector led to fewer instances of marked FGR, defined as fetal weight more than two standard deviations from the contemporaneous non growth restricted control mean, at term ${ }^{9}$. There was also a fall in ratio of the head (biparietal diameter, BPD) to abdominal size (abdominal circumference, AC) over gestation, which was significantly lower at term than control untreated FGR fetuses, and no different to non growth restricted control fetuses. There was no significant difference in the BPD measurements, suggesting that the "brain sparing" that is seen in this paradigm of fetal growth restriction had been ameliorated ${ }^{9}$.

The guinea pig is an appropriate model for human FGR and normal pregnancy as compared with other rodents, since their placentation is more similar to that of human; having a homologous 
process of trophoblast invasion ${ }^{10}$ and trophoblast cell proliferation ${ }^{11}$. In addition, they have a haemochorial type of placenta, as in humans ${ }^{12}$. Interventions such as periconceptual maternal nutrient restriction leads to asymmetric growth restriction with similar long term consequences to human FGR ${ }^{13}$. The long gestational age of 65-68 days in the guinea pig allows time for therapy to be administered in mid or late gestation and for an effect on fetal growth to be realized.

Therapeutic adenoviral vector delivery to adult guinea pigs has been described via the following injection routes: intradermal $1^{14}$, subcutaneous ${ }^{15}$, intramuscular ${ }^{16}$, intraarticular ${ }^{17}$, heart (left ventricle $)^{18}$ and intracochlear ${ }^{19}$. Senoo et al. detected the adenovirus vector in several fetal organs, after injecting vectors into the umbilical vein of late gestation guinea pig fetuses at laparotomy ${ }^{20}$; our aim however was to test different methods to achieve localized maternal gene delivery to the uterine arteries without spread to the fetus as a therapeutic approach to prevent FGR. We propose that maternal gene therapy localized to the uterine vasculature, producing an increase in uteroplacental perfusion, represents a solution to treating FGR, and this is currently being translated into the clinic in the EVERREST programme (www.everrest-fp7.eu). Our long term goal is to first evaluate if local maternal VEGF gene transfer to the uterine and radial arteries improves fetal growth in an FGR guinea pig model, and later in human pregnancies complicated by FGR. For this purpose, the experiments described were conducted with an adenovirus encoding a reporter gene $\beta$-galactosidase rather than VEGF, in order to be able to quickly stain the blood vessels and evaluate the extent of transgenic protein over-expression. We tested two local uteroplacental intravascular routes of administration and an external vascular transduction method combining vector with either PBS or pluronic gel. Pluronic gel is a thermolabile gel that is liquid when cold, but solidifies at body temperature. It has commonly been used for drug and viral delivery to a variety of vascular beds in pre-clinical and clinical studies and shown to be safe to administer, non-irritant and cytocompatible $\mathrm{e}^{21-24}$. 


\section{Methods}

Time-mated female Dunkin Hartley guinea pigs (Harlan Animal Research Laboratory, UK) were scanned at 20-30 days gestational age (dga) to confirm pregnancy, using a 7-10 $\mathrm{MHz}$ probe (Siemens, Bracknell, United Kingdom). A midline laparotomy was performed under general anaesthesia (diazepam 5mg/kg, atropine 0.5mg/kg, ketamine $40 \mathrm{mg} / \mathrm{kg}$; Hameln Pharmaceuticals, Gloucester, UK) at either $\sim 30 \mathrm{dga}$ or $\sim 45 \mathrm{dga}$, maintained by isoflurane inhalation (1.5-2.0\%) and an adenovirus vector encoding for a reporter $\beta$-galactosidase gene (Ad.LacZ, 1x10 ${ }^{10}$ viral particles per animal) was administered to the uterine and radial arteries by four different methods described below.

\section{Intra-vascular injection of the vector into the uterine arteries}

For direct injection into uterine arteries, the vessel (200-350 $\mu \mathrm{m}$ diameter) was located as it ran in the posterior surface of the mesometrium lying parallel and lateral to each uterine horn, and visualised using a light (KL 1500 LCD, Schott, Mainz, Germany) shone through the mesometrium from behind. A $3 \mathrm{~cm}$ section of the uterine artery within the posterior surface of the mesometrium was gently cleared of surrounding fat and exposed using a small straight vannas scissors (103102, John Weiss and Company, Milton Keynes, UK). The vessel proximal to the injection site was temporarily occluded using a microvascular clip (610195, Harvard Apparatus, Kent, UK) and the vector $\left(1 \times 10^{10}\right.$ viral particles of Ad.LacZ in a volume of $0.5-1.3 \mathrm{ml}$ PBS $)$ was then administered over approximately 1 minute using a custom made 34 Gauge needle mounted on a short $5 \mathrm{~cm}$ catheter attached to a $1 \mathrm{ml}$ syringe. Once the needle was removed, a second microvascular clip was placed over the injection site to compress the injection site, for a minimum of 3 minutes after which both occluders were removed and the vessel was carefully observed for bleeding. If necessary to achieve haemostasis, adrenaline (10 $\mu$, Hameln 
Pharmaceuticals, Gloucester, UK) was applied to the vessel for vasoconstriction. The injection procedure was then attempted in the uterine artery on the other horn.

\section{Intra-vascular injection of the vector into the internal iliac arteries}

The internal iliac artery has the uterine artery as its most distal branch situated approximately $3.5 \mathrm{~cm}$ from the origin of the internal iliac artery (Figure 1). For direct injection of the internal iliac artery, the vessel was identified as it runs with a neurovascular bundle along the pelvic side wall beneath the posterior aspect of the peritoneum at the level of the cervix. Careful dissection of the parietal peritoneum over the neurovascular bundle with a small straight vannas scissors revealed the prominent internal iliac vein, and the smaller sized artery $(200-250 \mu \mathrm{m}$ in diameter $)$ behind it, as it coursed transversely from its origin at the common iliac artery towards the cervical parametrium. A $1.5 \mathrm{~cm}$ portion of the artery was exposed, and the vessel was temporarily occluded using a microvascular clip, and the vector was administered distal to the occlusion over approximately 1 minute using a 34 Gauge needle as described before $\left(1 \times 10^{10}\right.$ viral particles in 1.0-1.5 ml of PBS). Following vector injection and removal of the needle, a second microvascular clip was placed over the injection site for 3 minutes. Both clips were then removed and the vessel was carefully observed for bleeding. Adrenaline was applied externally to the vessel if necessary, as described before, to achieve haemostasis. The injection procedure was then attempted on the opposite side internal iliac artery.

A successful injection of the uterine or internal iliac arteries was defined as the vector fluid seen moving down the vessel during injection. Failed injections were due to vector being inadvertently injected into the vessel wall, leaking out of the vessel or coming out of the other side of the vessel, opposite the injection site. In case of failed injections, 1-2 further attempts to inject the artery were made. A dissecting microscope (Discovery V8 Stereomicroscope, Carl Zeiss Ltd., Herts UK) was used to visualise vessels during dissection and intravascular injection. 


\section{External administration of the vector to the uterine arteries in PBS}

For external vascular administration of the vector in PBS, the vector $\left(1 \times 10^{10}\right.$ viral particles Ad.LacZ in a total volume of $0.5-1.0 \mathrm{ml}$ PBS bilaterally) was dribbled onto the exposed uterine artery within the mesometrial fat and the vessel was left still for 5 minutes before the uterus was subsequently mobilized. Previous experiments in sheep have demonstrated that a 5 minute contact period of the vector with the uterine artery is sufficient to result in significant transgenic protein expression ${ }^{7}$. During this 5 minute time period, if access to the uterine artery necessitated the uterus to be lifted out of the abdominal cavity, the uterine horn was kept warm and moist by wrapping it in a sterile gauze soaked with warm saline. No vessel occlusion was used in this technique. For radial artery gene transfer, the uterine horn on one side was moved across to expose the arteries as they ran within the uterine mesometrium. Sometimes it was not possible to transduce the uterine artery and radials at the same time because of difficulties in exposing all these vessels simultaneously. In such instances, the uterine artery was transduced first and after a 5 minute wait, the radials were exposed and transduced. The procedure was repeated on the contralateral side.

\section{External administration of the vector to the uterine arteries in pluronic gel}

For external vascular administration of the vector in Pluronic gel, the vector was combined with a freshly made thermo-sensitive polymer gel (Pluronic F-127) that is liquid when cold, but gelatinizes when brought to room temperature. A 25\% (w/v) solution of Pluronic F-127 (P2443, Sigma Aldrich, USA) was prepared by dissolving the powder in cold double distilled de-ionized water the day before surgery. The solution was placed on a rotating platform overnight at $4^{\circ} \mathrm{C}$ to ensure the polymer was homogenously mixed. Immediately before administration, the vector (Ad.LacZ, $1 \times 10^{10}$ viral particles) was reconstituted in $1 \mathrm{ml}$ of Pluronic gel and mixed by gently pipetting up and down, on ice. After exposing the uterine artery as it ran within the mesometrium, 
the vector-pluronic gel combination was dribbled onto the external surface of the uterine artery using a $1 \mathrm{ml}$ micro-pipette, and left to solidify for 5 minutes. Similarly the radial arteries were then transduced for a further 5 minutes. No vessel occlusion was used in this technique. The procedure was then repeated on the contralateral side.

After vector administration (by either of the four methods described above), the abdomen was closed in layers. The rectus sheath was closed with continuous 2-0 Vicryl with Tapercut needle (Ethicon, St. Stevens-Woluwe, Belgium) to prevent herniation of the abdominal contents. The subcutaneous tissue and skin were closed with continuous 2-0 Vicryl with Tapercut needle (Ethicon, St. Stevens-Woluwe, Belgium) and continuous 2-0 Vicryl with Cutting needle (Ethicon, St. Stevens-Woluwe, Belgium) respectively. A $10 \%$ solution of lidocaine hydrochloride $(0.5 \mathrm{ml})$ was administered subcutaneously just before closing the skin to provide local anaesthesia.

Because there are only few antibiotics that are well-tolerated in guinea pigs, and many of those commonly used ones such as Penicillin-streptomycin kill their symbiotic gut flora, no antibiotics were administered intra-peritoneally prior to closing the incision. Following surgeries and recovery, animals were monitored for signs of miscarriage.

\section{Post-mortem examination}

Scheduled post-mortem examination was performed 4-7 days after the administration of the vector, unless necessitated earlier by miscarriage or maternal distress. Survival data is presented on each route of injection. All procedures on animals were conducted in accordance with UK Home Office regulations and the Guidance for the Operation of Animals (Scientific Procedures Act 1986).

The fetuses were carefully evaluated for signs of miscarriage. Widespread sampling of maternal and fetal tissues was performed (maternal organs including uterine and radial arteries, uterus, 
cervix, placentae, bladder, pelvic muscles, as well as maternal and fetal liver, heart, kidney, lung, adrenal, spleen and gonad). A detailed post mortem examination and microbiological culture of tissues was performed on tissues of animals that died after surgery to determine the cause of death.

\section{Transgenic protein expression}

For X-gal histochemistry tissue samples were placed in $100 \%$ ethanol at post-mortem examination and fixed for at least 2 hours before washing with PBS. Samples were then incubated in X-gal staining solution overnight in a rotary shaker protected from light, as described earlier ${ }^{7}$. Stained samples were then washed in PBS and photographed with a camera attached to a microscope (Discovery V8 Stereomicroscope, Carl Zeiss Ltd., Herts UK and Canon Powershot A620 digital camera).

\section{Enzyme-Linked Immuno Sorbent Assay (ELISA)}

$\beta$-galactosidase levels in the UtAs, radial arteries, placentae, uterus, maternal and fetal liver, heart, kidney, brain, spleen and gonads were determined by ELISA using a commercially available assay kit (Boehringer Mannheim, Mannheim, Germany) as previously described ${ }^{7}$.

\section{Statistics}

Results are presented as means \pm SEM. Statistical analysis was performed using GraphPad Prism Version 5 by using one-way ANOVA (Kruskal-Wallis Test), to compare $\beta$-galactosidase levels observed using the four different routes of administration. 


\section{Results}

The success rate of the four different methods of administration of adenoviral vectors to the uterine and radial arteries of pregnant guinea pigs is shown in Table 1. Maternal survival was described in terms of survival to scheduled post mortem examination 4-7 days after surgery.

\section{Uterine artery injections}

Injection of the uterine arteries of pregnant guinea-pigs at 30 dga was hampered by technical difficulties due to the small size of the vessels at mid-gestation $(150-250 \mu \mathrm{m})$ preventing reliable and safe injection ( $\mathrm{n}=2$ out of $8 ; 25 \%$ success rate, $50 \%$ survival). Deferring injection until $3 / 4$ through gestation (45dga) was more feasible with successful injection seen in $44 \%$ of attempts ( 7 out of 16 uterine arteries, $n=8$ dams), even though the vessels were still small $(300-400 \mu m$ diameter). The low success rate is attributed to difficulties dissecting out and injecting the uterine artery, which is deeply embedded in fat and is unsupported by the surrounding tissue. In addition it is likely that even in those apparently successfully injected vessels, much of the vector leaked out of the vessel and behaved similarly to the external administration of vector with PBS leading to poor local gene transfer. There were two maternal deaths, one during surgery that was ascribed to the long anaesthetic time ( $>4 \mathrm{hrs}$ ) and one within 24 hours of the surgery that was due to sepsis. There was no fetal loss in mothers who survived the procedure.

Uterine artery injection $(500 \mu l$ vector) resulted in gene transfer to the cervical end of the uterine arteries and the cervical end radial arteries. Increasing the injected vector volume from $0.5 \mathrm{ml}$ to $1.0 \mathrm{ml}$ resulted in gene transfer also to the radial arteries supplying the middle fetus (of three on one side). However, even the maximum volume that was injected $(1.3 \mathrm{ml})$ was not successful in transducing the ovarian end of the uterine artery and radial arteries supplying the fetus at the 
ovarian end of the uterine horns. The maternal liver, uterus and placentae were marginally stained. No staining of the fetuses or placentae was seen.

\section{2) Injection of internal iliac arteries}

Internal iliac artery injection was successful in all 12 vessels ( $n=6$ dams, 45-47 dga) although multiple attempts were required in each of the 12 vessels. Maternal survival was $66 \%$ (4 out of 6), fetal survival was $100 \%$ in those dams that survived. The deaths occurred only in the dams that received $1.5 \mathrm{ml}$ vector, which was probably related to bleeding following the multiple (5-6) attempts that were needed on each side to inject the full volume of vector, rather than vector toxicity.

Administration of Ad.LacZ into the internal iliac artery resulted in positive X-gal staining in the internal iliac artery, maternal liver, pelvic muscles, bladder and cervix. There was no expression in the uterine/radial arteries, the fat around the uterine arteries, the placentae or fetuses. Increasing the volume of vector injected did not lead to positive expression in the uterine artery.

\section{3) External transduction of uterine and radial arteries with vector in PBS}

External transduction of the exposed uterine artery by the vector in PBS was technically easy to perform, less traumatic compared with the intravascular injection methods with a good survival rate (5 out of 6 dams survived, no fetal losses in surviving dams). The only maternal death happened during a long 4 hour surgery where exposure of the uterine artery was technically difficult and led to bleeding.

External administration of the vector to the uterine and radial arteries resulted not only in positive expression in all the vessels on which the vector had been applied, but also in the fat surrounding 
the uterine arteries. All radial arteries stained positively, as well as the maternal liver and uterus. Although there was slight staining in the placentae, there was none detected in the fetuses.

\section{4) External Administration of Vector-Pluronic Gel Combination}

Administration of the vector in combination with thermolabile pluronic gel was technically straightforward, shortening the duration of the surgery by more than half (from 3-4 hours to only 1 hour) with successful gene transfer in $91.7 \%$ of cases ( $n=11$ out of 12 vessels, $n=6$ dams). Fetal survival was $100 \%$ in the dams that survived successfully. In one case it was not possible to administer the vector to the right uterine artery because the vessel was very small and consequently difficult to visualize within the fat pad. This dam miscarried the next morning, probably due to excessive manipulation of the uterine horn, which had to be exposed for long periods of time during surgery. There was no evidence of peritoneal inflammation, bleeding or infection at postmortem examination.

Administration of Ad.LacZ using Pluronic gel as a vehicle led to very high levels of local transgenic protein expression in the uterine and radial arteries to which the vector had been applied, not only on the external surface but inner luminal surface as well. The liver and uterus had minimal $\beta$-galactosidase expression in one animal and did not stain positively in any of the other animals, when incubated in the X-gal staining solution overnight. All other maternal and fetal tissues sampled (placentae, heart, kidney, lung, gonad, bladder, brain and adrenal) did not show any positive staining with the X-gal staining solution.. Representative pictures of X-gal staining in the uterine arteries, after the different methods of administration, are shown in Figure 2.

\section{Measurment of $\beta$-galactosidase levels by ELISA}


Quantitative analysis of $\beta$-galactosidase expression in tissues using ELISA found that the most effective route of administration was combination of pluronic gel and vector, yielding the highest mean levels in samples collected from uterine artery $(\mathrm{p}=0.0048, \mathrm{n}=4$ vessels for each route of administration, one-way ANOVA). Administration of Ad.LacZ via uterine artery injection yielded similar results to external route of administration using PBS and Ad.LacZ vector combination. After internal iliac artery injection, no $\beta$-galactosidase expression was detected in uterine or radial arteries (Table 2, Figure 3).

$\beta$-galactosidase expression was also quantified in the fetal tissues (kidney, gonad, muscle, bone marrow, thymus and amniotic fluid). The highest $\beta$-galactosidase concentration was observed in amniotic fluid (45 $\mathrm{pg} / \mathrm{mg}$ ) after administering vector externally to the uterine artery and radial arteries in PBS. The second highest value of $\beta$-galactosidase was observed in bone marrow (14 $\mathrm{pg} / \mathrm{mg}$ ) after uterine artery injection with the vector. Placental $\beta$-galactosidase expression was detected only in the animals wherein external transduction of Ad.LacZ was performed using PBS as a vehicle, and was quantified to be $57 \mathrm{pg} / \mathrm{mg}$. None of the other tissues showed positive $\beta$ galactosidase expression by any of the routes of administration. 


\section{Discussion}

In this study we have demonstrated that the most effective method of gene transfer to the pregnant guinea pig uterine and radial arteries is external administration of the vector in combination with pluronic gel as a vehicle. Moreover, application of the vector by this technique is technically straightforward and atraumatic as an intravascular injection is not required. The delivery method achieved localized gene transfer restricted only to the uterine and radial arteries, with no spread of vector to other maternal and fetal tissues. Since pluronic gel becomes solid immediately after administration, due to its characteristic property of reversible thermogelation, there is minimal spread of the vector to other organs. In particular with relevance to maternal uteroplacental gene transfer, external vector administration using pluronic gel was safe for the developing fetus as there was no vector spread to fetal tissues. In addition external application of adenovirus either in pluronic gel or PBS achieved $84 \%-100 \%$ fetal survival, in contrast to direct intraplacental adenoviral administration, that only achieved fetal survival rates of $30 \%-42 \%{ }^{25}$. Our results indicate that using a localized pluronic gel based depot of slow viral vector release facilitates greater transgene expression in comparison to other techniques, when direct vascular injection may be technically challenging. Pluronic gel has been used to deliver Ad.LacZ to the carotid artery of rats. Five days post-infection, the presence of the $\beta$-gal transgene was visualized throughout the arterial wall ${ }^{21}$. The combination of vector with pluronic gel has been used as a vehicle for virus-mediated gene delivery for a number of indications, but not previously to the vessels of the utero-placental circulation. Poloxamer gels display low toxicity at therapeutically beneficial doses and do not increase serum triglycerides and cholesterol in animal models ${ }^{26}$. Clinical uses of pluronic F-127 have included the controlled delivery of drugs to the eye ${ }^{27}$, nasal passage as well as parenteral and subcutaneous administration ${ }^{28}$. Pluronic gels have been used as

a vehicle for local adenoviral gene delivery previously in pre-clinical and clinical studies ${ }^{21,23,29}$ and shown to be safe to administer. Depending on the concentration of the gel, it may last from a 
few hours to a few days, undergoing slow dissolution in the body fluid. Pluronics have been shown to be non-irritant, non-inflammatory and cytocompatible with many different cell types ${ }^{30}$. They are primarily cleared from the body via the renal route in the form of unimers ${ }^{31}$. They increase the transduction efficiency of adenoviral vector delivery to vascular cells in vivo compared to $\mathrm{PBS}^{24}$, probably because of the slower kinetics associated with the longer term vector release from the gel or the closer proximity of viral vector to the cell surface. Because of the thermo-responsive behaviour of pluronic F-127, the gel is delivered in vivo over a short period of time before solidification.

In our study, a higher concentration of $\beta$-galactosidase expression was observed in the radial arteries compared with the uterine arteries. This might be due to the finding that the uterine arteries are deeply embedded in fat, whilst the radial arteries are only covered by a thin mesentery, allowing more effective diffusion of the viral vector through the vessel wall. External administration of Ad.LacZ vector combined with PBS led to low transgenic protein expression in the vessels, probably due to the low viscosity of PBS solution, which allowed it to spread from the target vessel to surrounding tissues. Although the uterine and radial arteries were left still for 5 minutes after application of the PBS Ad.LacZ vector combination, the fluid was seen to dribble down into the pelvic cavity. Our previous work in sheep demonstrates that contact of the vector with the uterine artery for 5 minutes is sufficient to result in significant transgenic protein expression $^{7}$.

Intra-arterial administration of vector into the uterine arteries of pregnant guinea pigs only led to partial transduction of the utero-placental circulation that was limited to those vessels closest to the injection site. Increasing the volume of vector injected did not result in transduction of the furthest zones. Similarly, in an angiographic study to examine the vascular anatomy of guinea pig uterine arteries, contrast medium injected into the uterine artery in vivo failed to enter the most distal radial arteries and placentae $e^{32}$, probably because they are preferentially served by the 
ovarian arteries. Vector injection into the ovarian arteries was considered, but because they were of even smaller diameter than the uterine arteries it was not attempted. Delivery into the uterine artery using interventional radiology was considered, since guinea pig uterine arteries have a sufficiently wide calibre at mid-gestation to be easily injectable by angiographic guided catheter ${ }^{25}$. We were concerned however that trauma to the vessel internally would result in a high rate of miscarriage.

Although injection of internal iliac artery was feasible, gene transfer to the uterine arteries was not achieved and instead, there was gene transfer observed preferentially to the guinea-pig pelvis. This was not unexpected since the arteries supplying the pelvic musculature and organs are the first three branches of the internal iliac artery, which suggests that the vector has been redirected to those vessels by haemodynamic forces. Moreover, the technique was associated with significant fetal and maternal loss, most probably because of the long anaesthetic time required to complete the procedure.

We observed that intra-vascular administration of Ad.LacZ as well as external administration in PBS resulted in strong liver transduction. Previous studies of adenovirus gene transfer in the guinea pig found a strong liver tropism, most likely due to the high density of coxsackievirus and adenovirus receptor on hepatocytes ${ }^{33-36}$. We did not observe this liver tropism in dams which received Ad.LacZ in pluronic gel, most probably because the gel confined the viral particles to the site of administration with minimal spread to other tissues and organs.

The efficacy of maternal uteroplacental VEGF gene therapy to treat FGR has been tested in growth restricted sheep where the large vessel size permits safe direct intravascular injection ${ }^{9}$. It was important for translation into the clinic to demonstrate efficacy for improved fetal growth in a second animal model of FGR, and we have chosen to use the guinea pig for reasons described previously. Safe and high level local gene transfer was not achieved using direct intravascular 
injection due to the technical difficulties encountered, but was demonstrated using external administration with pluronic gel. Current studies are investigating the efficacy of this treatment in the maternal nutrient restricted guinea pig that has asymmetrical FGR, and will include examination of the mechanism of action of the intervention. For human trials, we propose using an interventional radiology technique to deliver vector into the uterine artery that is currently applied in the clinic for embolization of uterine fibroids ${ }^{37}$ or prevention of postpartum haemorrhage ${ }^{38}$.

Direct adenovirus vector injection into the uterine arteries of pregnant sheep is safe and leads to high local transgenic protein expression with minimal maternal and fetal mortality or morbidity ${ }^{7-}$ ${ }^{9,39}$. Injection of the uterine artery in the rabbit can be achieved using catheter-mediated injection under angiographic control ${ }^{40}$. This leads to high transduction efficiency in the uterine artery and placental trophoplastic cells. The much smaller diameter of the guinea pig UtA compared to the sheep UtA makes its injections more challenging and more prone to complications.

In summary, we have shown in the pregnant guinea pig that administration of recombinant adenoviruses using thermolabile pluronic gel as a vehicle leads to robust transgenic protein expression in the utero-placental vessels, both on the adventitial and luminal surfaces. There is no vector expression in other maternal and fetal tissues. Surgery time was shorter when compared to alternative intravascular injection methods, thereby lowering the risk of morbidity and mortality. Our findings suggest that vector administration using Pluronic F-127 as a vehicle is the optimum method of gene targeting to the uteroplacental blood vessels of pregnant guinea pigs. 


\section{Acknowledgements}

Funding for the study was received from the following sources: Action Medical Research,

UCL/UCLH NIHR Comprehensive Biomedical Research Centre (ALD, DMP), The British Heart

Foundation (ICZ), Ark Therapeutics Oy, Kuopio, Finland (supplied adenovirus vectors free of charge). VM is a Dorothy Hodgkins Postgraduate Awardee. 


\section{REFERENCES}

1. McIntire DD, Bloom SL, Casey BM, Leveno KJ. Birth weight in relation to morbidity and mortality among newborn infants. The New England journal of medicine. 1999;340(16):1234-1238.

2. Doctor BA, O'Riordan MA, Kirchner HL, Shah D, Hack M. Perinatal correlates and neonatal outcomes of small for gestational age infants born at term gestation. American journal of obstetrics and gynecology. 2001;185(3):652-659.

3. Jarvis S, Glinianaia SV, Torrioli MG, et al. Cerebral palsy and intrauterine growth in single births: European collaborative study. Lancet. 2003;362(9390):1106-1111.

4. Blair E, Stanley F. Intrauterine growth and spastic cerebral palsy. I. Association with birth weight for gestational age. American journal of obstetrics and gynecology. 1990;162(1):229-237.

5. Thornton JG, Hornbuckle J, Vail A, Spiegelhalter DJ, Levene M, group Gs. Infant wellbeing at 2 years of age in the Growth Restriction Intervention Trial (GRIT): multicentred randomised controlled trial. Lancet. 2004;364(9433):513-520.

6. Barker DJ. Fetal growth and adult disease. British journal of obstetrics and gynaecology. 1992;99(4):275-276.

7. David AL, Torondel B, Zachary I, et al. Local delivery of VEGF adenovirus to the uterine artery increases vasorelaxation and uterine blood flow in the pregnant sheep. Gene therapy. 2008;15(19):1344-1350.

8. Mehta V, Abi-Nader KN, Peebles DM, et al. Long-term increase in uterine blood flow is achieved by local overexpression of VEGF-A(165) in the uterine arteries of pregnant sheep. Gene therapy. 2012;19(9):925-935.

9. Carr DJ, Wallace JM, Aitken RP, et al. Uteroplacental adenovirus vascular endothelial growth factor gene therapy increases fetal growth velocity in growth-restricted sheep pregnancies. Human gene therapy. 2014;25(4):375-384.

10. Carter AM, Enders AC, Jones $\mathrm{CJ}$, et al. Comparative placentation and animal models: patterns of trophoblast invasion -- a workshop report. Placenta. 2006;27 Suppl A:S3033.

11. Mess A. The Guinea pig placenta: model of placental growth dynamics. Placenta. 2007;28(8-9):812-815.

12. Carter AM. Animal models of human placentation--a review. Placenta. 2007;28 Suppl A:S41-47.

13. Roberts $\mathrm{CT}$, Sohlstrom A, Kind KL, et al. Maternal food restriction reduces the exchange surface area and increases the barrier thickness of the placenta in the guinea-pig. Placenta. 2001;22(2-3):177-185.

14. Byun J, Heard JM, Huh JE, et al. Efficient expression of the vascular endothelial growth factor gene in vitro and in vivo, using an adeno-associated virus vector. Journal of molecular and cellular cardiology. 2001;33(2):295-305.

15. Wang $D$, Hevey M, Juompan LY, et al. Complex adenovirus-vectored vaccine protects guinea pigs from three strains of Marburg virus challenges. Virology. 2006;353(2):324332.

16. Chen $\mathrm{W}$, Liu M, Jiao $\mathrm{Y}$, et al. Adenovirus-mediated RNA interference against foot-andmouth disease virus infection both in vitro and in vivo. Journal of virology.

2006;80(7):3559-3566. 
17. Santangelo KS, Baker SA, Nuovo G, Dyce J, Bartlett JS, Bertone AL. Detectable reporter gene expression following transduction of adenovirus and adeno-associated virus serotype 2 vectors within full-thickness osteoarthritic and unaffected canine cartilage in vitro and unaffected guinea pig cartilage in vivo. Journal of orthopaedic research : official publication of the Orthopaedic Research Society. 2010;28(2):149-155.

18. Kapoor N, Liang W, Marban E, Cho HC. Direct conversion of quiescent cardiomyocytes to pacemaker cells by expression of Tbx18. Nature biotechnology. 2013;31(1):54-62.

19. Li Duan M, Bordet T, Mezzina M, Kahn A, Ulfendahl M. Adenoviral and adeno-associated viral vector mediated gene transfer in the guinea pig cochlea. Neuroreport. 2002;13(10):1295-1299.

20. Senoo M, Matsubara Y, Fujii K, et al. Adenovirus-mediated in utero gene transfer in mice and guinea pigs: tissue distribution of recombinant adenovirus determined by quantitative TaqMan-polymerase chain reaction assay. Molecular genetics and metabolism. 2000;69(4):269-276.

21. laccarino G, Smithwick LA, Lefkowitz RJ, Koch WJ. Targeting Gbeta gamma signaling in arterial vascular smooth muscle proliferation: a novel strategy to limit restenosis. Proceedings of the National Academy of Sciences of the United States of America. 1999;96(7):3945-3950.

22. Khurana R, Zhuang Z, Bhardwaj S, et al. Angiogenesis-dependent and independent phases of intimal hyperplasia. Circulation. 2004;110(16):2436-2443.

23. Mallawaarachchi CM, Weissberg PL, Siow RC. Smad7 gene transfer attenuates adventitial cell migration and vascular remodeling after balloon injury. Arteriosclerosis, thrombosis, and vascular biology. 2005;25(7):1383-1387.

24. Feldman $\sqcup$, Pastore $\mathrm{CJ}$, Aubailly N, et al. Improved efficiency of arterial gene transfer by use of poloxamer 407 as a vehicle for adenoviral vectors. Gene therapy. 1997;4(3):189198.

25. Xing A, Boileau P, Cauzac M, Challier JC, Girard J, Hauguel-de Mouzon S. Comparative in vivo approaches for selective adenovirus-mediated gene delivery to the placenta. Human gene therapy. 2000;11(1):167-177.

26. Blonder JM, Baird L, Fulfs JC, Rosenthal GJ. Dose-dependent hyperlipidemia in rabbits following administration of poloxamer 407 gel. Life sciences. 1999;65(21):PL261-266.

27. El-Kamel AH. In vitro and in vivo evaluation of Pluronic F127-based ocular delivery system for timolol maleate. International journal of pharmaceutics. 2002;241(1):47-55.

28. Barichello JM, Morishita M, Takayama K, Nagai T. Absorption of insulin from pluronic F127 gels following subcutaneous administration in rats. International journal of pharmaceutics. 1999;184(2):189-198.

29. Khurana R, Martin JF, Zachary I. Gene therapy for cardiovascular disease: a case for cautious optimism. Hypertension. 2001;38(5):1210-1216.

30. Akash MS, Rehman K, Chen S. Pluronic F127-Based Thermosensitive Gels for Delivery of Therapeutic Proteins and Peptides. Polymer Reviews. 2014;54(4):573-597.

31. Batrakova EV, Li S, Li Y, Alakhov VY, Elmquist WF, Kabanov AV. Distribution kinetics of a micelle-forming block copolymer Pluronic P85. J Control Release. 2004;100(3):389-397.

32. Egund N, Carter AM. Uterine and placental circulation in the guinea-pig: an angiographic study. Journal of reproduction and fertility. 1974;40(2):401-410.

33. Alemany R, Curiel DT. CAR-binding ablation does not change biodistribution and toxicity of adenoviral vectors. Gene therapy. 2001;8(17):1347-1353. 
34. Einfeld DA, Schroeder R, Roelvink PW, et al. Reducing the native tropism of adenovirus vectors requires removal of both CAR and integrin interactions. Journal of virology. 2001;75(23):11284-11291.

35. Kass-Eisler A, Falck-Pedersen E, Elfenbein DH, Alvira M, Buttrick PM, Leinwand LA. The impact of developmental stage, route of administration and the immune system on adenovirus-mediated gene transfer. Gene therapy. 1994;1(6):395-402.

36. Leissner $P$, Legrand $V$, Schlesinger $Y$, et al. Influence of adenoviral fiber mutations on viral encapsidation, infectivity and in vivo tropism. Gene therapy. 2001;8(1):49-57.

37. Gupta JK, Sinha A, Lumsden MA, Hickey M. Uterine artery embolization for symptomatic uterine fibroids. Cochrane Database Syst Rev. 2012;5:CD005073.

38. Delotte J, Novellas S, Koh C, Bongain A, Chevallier P. Obstetrical prognosis and pregnancy outcome following pelvic arterial embolisation for post-partum hemorrhage. European journal of obstetrics, gynecology, and reproductive biology. 2009;145(2):129132.

39. Mehta $\mathrm{V}$, Abi-Nader KN, Shangaris $\mathrm{P}$, et al. Local over-expression of VEGF-DDeltaNDeltaC in the uterine arteries of pregnant sheep results in long-term changes in uterine artery contractility and angiogenesis. PloS one. 2014;9(6):e100021.

40. Heikkila A, Hiltunen MO, Turunen MP, et al. Angiographically guided utero-placental gene transfer in rabbits with adenoviruses, plasmid/liposomes and plasmid/polyethyleneimine complexes. Gene therapy. 2001;8(10):784-788. 


\section{Figure Legends}

Figure 1 - Diagram of the internal iliac artery and its branches in the guinea pig - cranial gluteal (supply the dorsolateral wall of pelvis), internal pudendal artery (supplying the external genitalia), inferior vesical artery (supplying the lower bladder and also forming the middle rectal artery supplying the rectum), vaginal artery and uterine artery, which is quite some way along the internal iliac artery. The injection sites on the uterine and internal iliac arteries have been marked.

Figure 2 - X-gal staining of guinea pig uterine and radial arteries 4-7 days after Ad.LacZ administration by one of the following different routes (A) direct injection into the uterine artery; (B) external administration in PBS; (C) external administration in pluronic gel - uterine artery; (D) external administration in pluronic gel - radial artery; (E) external administration in pluronic gel - uterine artery (luminal/inside view). Even though direct injection into the uterine artery led to some expression of $\beta$-galactosidase, it was less than that achieved via external administration of the vector. Maximum local tissue transduction was achieved when Ad.LacZ was administered using pluronic gel as a vehicle. Scale bar $=150 \mu \mathrm{m}$

Figure 3 - Quantification of protein levels of $\beta$-galactosidase by ELISA in the uterine and radial arteries following the different routes of administration. 
Figure 1

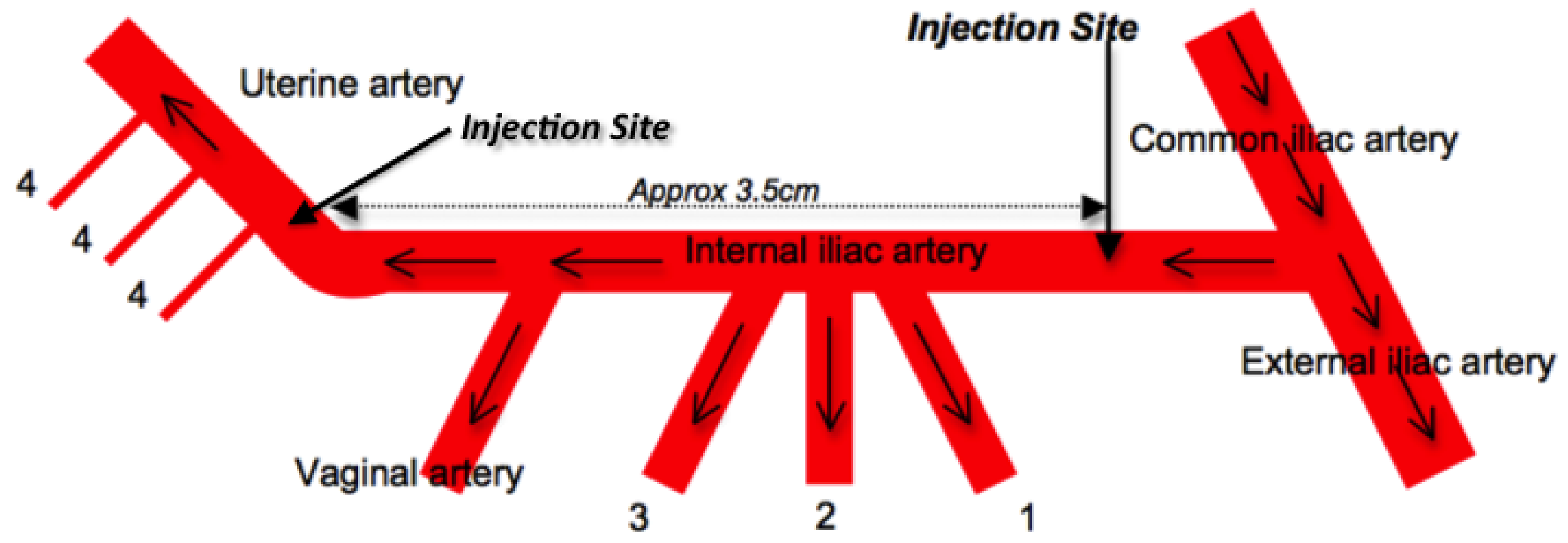

1. Cranial gluteal artery

2. Internal pudendal artery

3. Vesical artery

4. Radial artery 
Figure 2
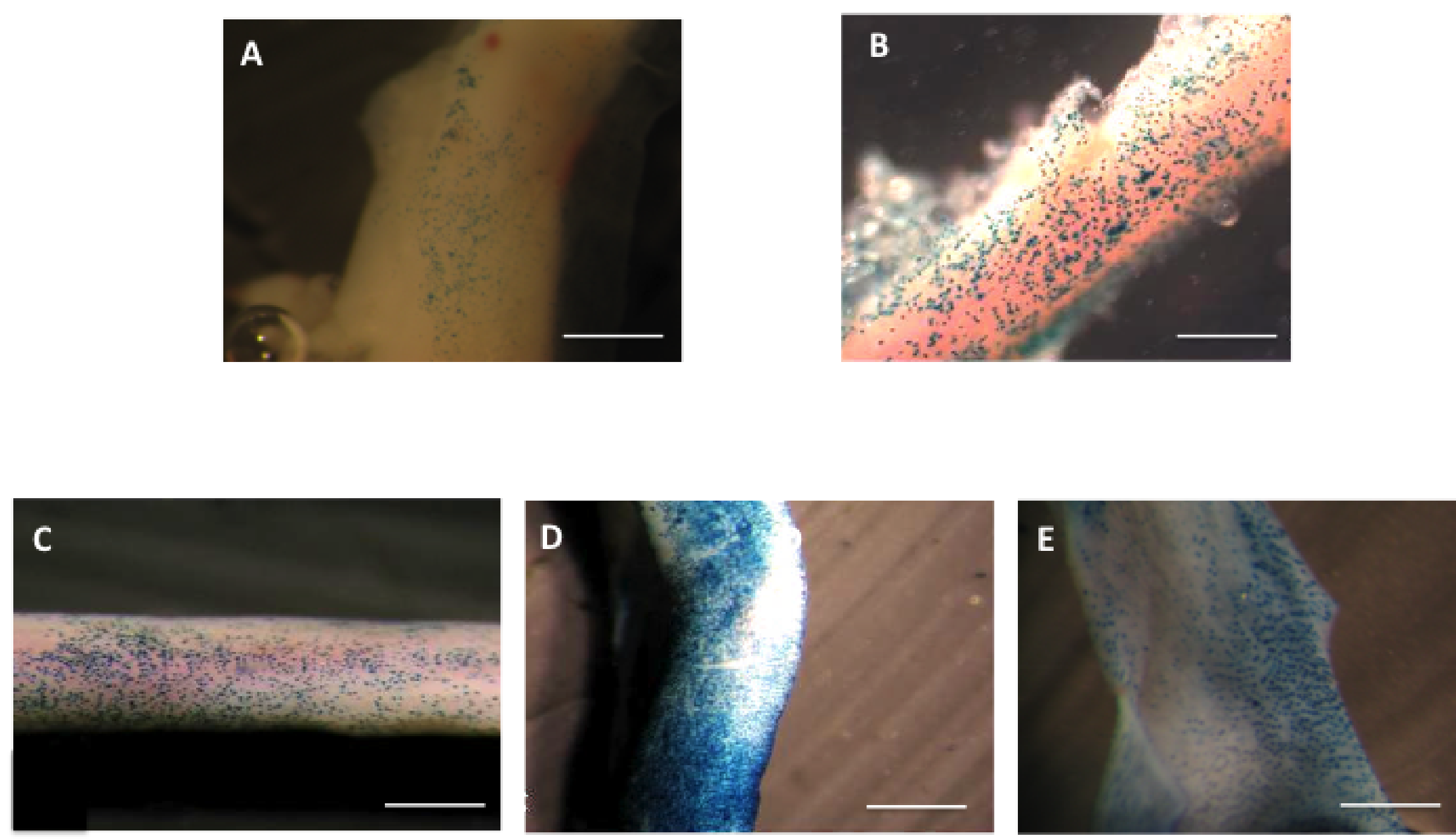
Figure 3

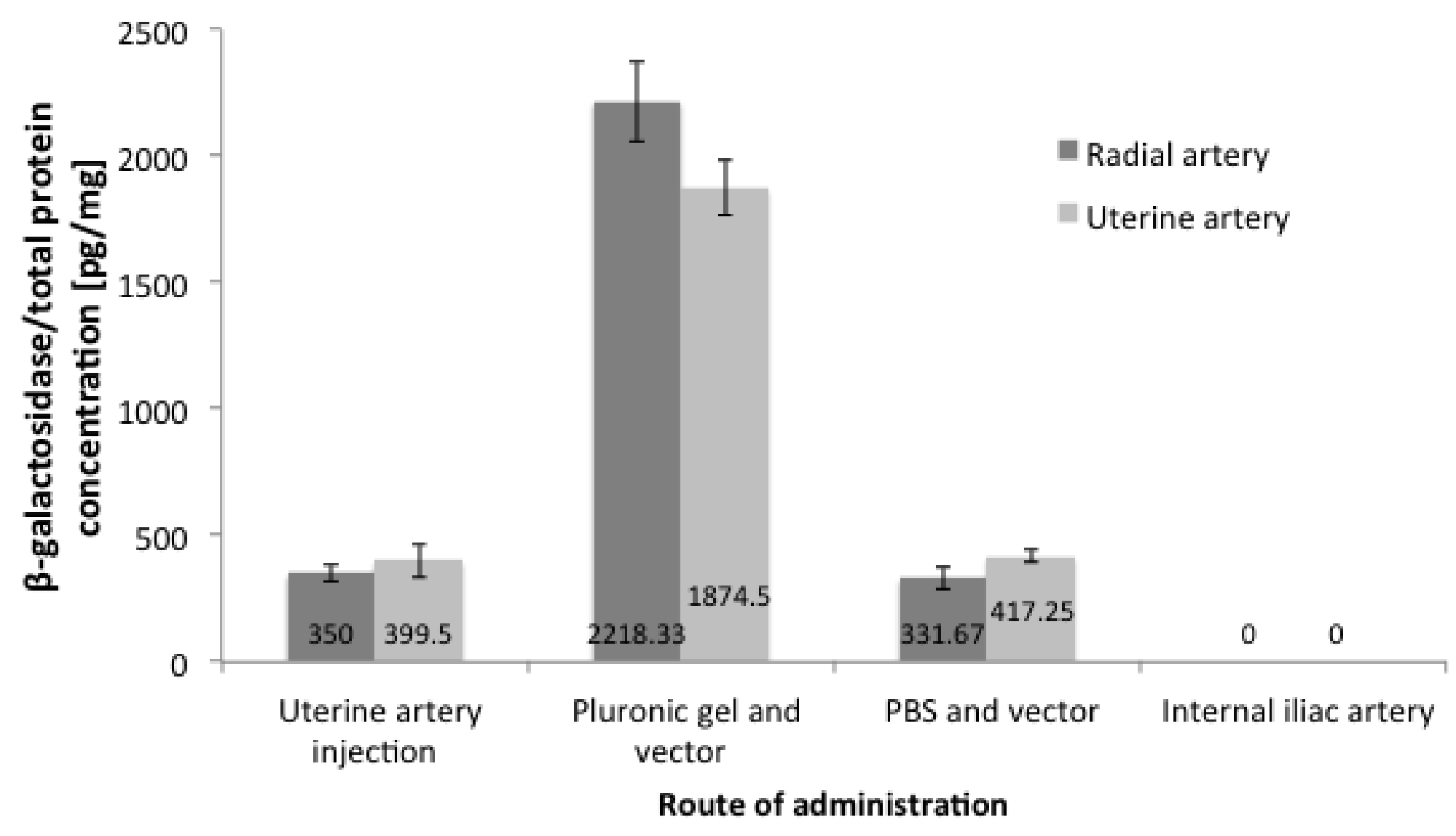


Table 1 - Surgical parameters using the different gene delivery techniques. Ad.LacZ was administered to the uterine and radial arteries of pregnant guinea pigs at 40-45 days of gestation, by one of the methods described in the table. The outcome of the surgery (in terms of maternal survival to scheduled post mortem examination 4-7 days after surgery) and the number of injection attempts required are summarised below.

\begin{tabular}{|c|c|c|c|}
\hline $\begin{array}{c}\text { Method of Ad.LacZ vector } \\
\text { administration }\end{array}$ & $\begin{array}{c}\text { Maternal } \\
\text { Survival }\end{array}$ & $\begin{array}{c}\text { Time taken to } \\
\text { inject }\end{array}$ & $\begin{array}{c}\text { Total number of } \\
\text { injection attempts } \\
\text { required (average) }\end{array}$ \\
\hline Uterine artery injection & $\begin{array}{c}6 / 8 \\
(75 \%)\end{array}$ & $2-2.5 \mathrm{hrs}$ & 4 \\
\hline Internal iliac injection & $\begin{array}{c}4 / 6 \\
(66 \%)\end{array}$ & $2-2.5 \mathrm{hrs}$ & 5 \\
\hline $\begin{array}{c}\text { External administration in } \\
\text { PBS }\end{array}$ & $\begin{array}{c}5 / 6 \\
(83.3 \%)\end{array}$ & $<1 \mathrm{hr}$ & 1 \\
\hline $\begin{array}{c}\text { External administration in } \\
\text { pluronic gel }\end{array}$ & $\begin{array}{c}5 / 6 \\
(83.3 \%)\end{array}$ & $<1 \mathrm{hr}$ & \\
\hline
\end{tabular}


Table 2 - Efficiency of gene transfer after administration of the vector using different routes. Tissue samples were collected at post-mortem examination and stained overnight in X-gal staining solution. Photomicrographs of the stained samples were carefully examined by a single observer who was blinded to the route of administration. The degree of blue staining, if any, was compared amongst all stained images and denoted as ' + ' indicating least staining and '++++' denoting maximum staining.

\begin{tabular}{|c|c|c|c|c|c|c|}
\hline $\begin{array}{l}\text { Method of } \\
\text { administration }\end{array}$ & \multicolumn{7}{|c|}{$\begin{array}{c}\text { Efficiency of gene transfer } \\
\text { artery }\end{array}$} & $\begin{array}{l}\text { Radial } \\
\text { artery }\end{array}$ & Mat. Liver & Mat. Lung & Uterus & Placenta \\
\hline $\begin{array}{c}\text { Uterine artery } \\
\text { injection }\end{array}$ & ++ & - & +++ & - & + & - \\
\hline $\begin{array}{c}\text { Internal iliac } \\
\text { injection }\end{array}$ & - & - & +++ & - & - & - \\
\hline $\begin{array}{c}\text { External } \\
\text { administration in } \\
\text { PBS }\end{array}$ & ++ & +++ & +++ & - & ++ & + \\
\hline $\begin{array}{c}\text { External } \\
\text { administration in } \\
\text { pluronic gel }\end{array}$ & ++++ & ++++ & - & - & - & - \\
\hline
\end{tabular}

